\title{
Thermal-Acoustic Analysis of a Metallic Integrated Thermal Protection System Structure
}

\author{
Marlana N. Behnke, ${ }^{1}$ Anurag Sharma ${ }^{2}$ \\ University of Florida, Gainesville, FL, 32611 \\ Adam Przekop ${ }^{3}$ \\ Analytical Services and Materials, Inc., Hampton, VA, 23666 \\ and \\ Stephen A. Rizzi ${ }^{4}$ \\ NASA Langley Research Center, Hampton, VA, 23681
}

\begin{abstract}
A study is undertaken to investigate the response of a representative integrated thermal protection system structure under combined thermal, aerodynamic pressure, and acoustic loadings. A two-step procedure is offered and consists of a heat transfer analysis followed by a nonlinear dynamic analysis under a combined loading environment. Both analyses are carried out in physical degrees-of-freedom using implicit and explicit solution techniques available in the Abaqus commercial finite-element code. The initial study is conducted on a reduced-size structure to keep the computational effort contained while validating the procedure and exploring the effects of individual loadings. An analysis of a full size integrated thermal protection system structure, which is of ultimate interest, is subsequently presented. The procedure is demonstrated to be a viable approach for analysis of spacecraft and hypersonic vehicle structures under a typical mission cycle with combined loadings characterized by largely different time-scales.
\end{abstract}

\section{Nomenclature}
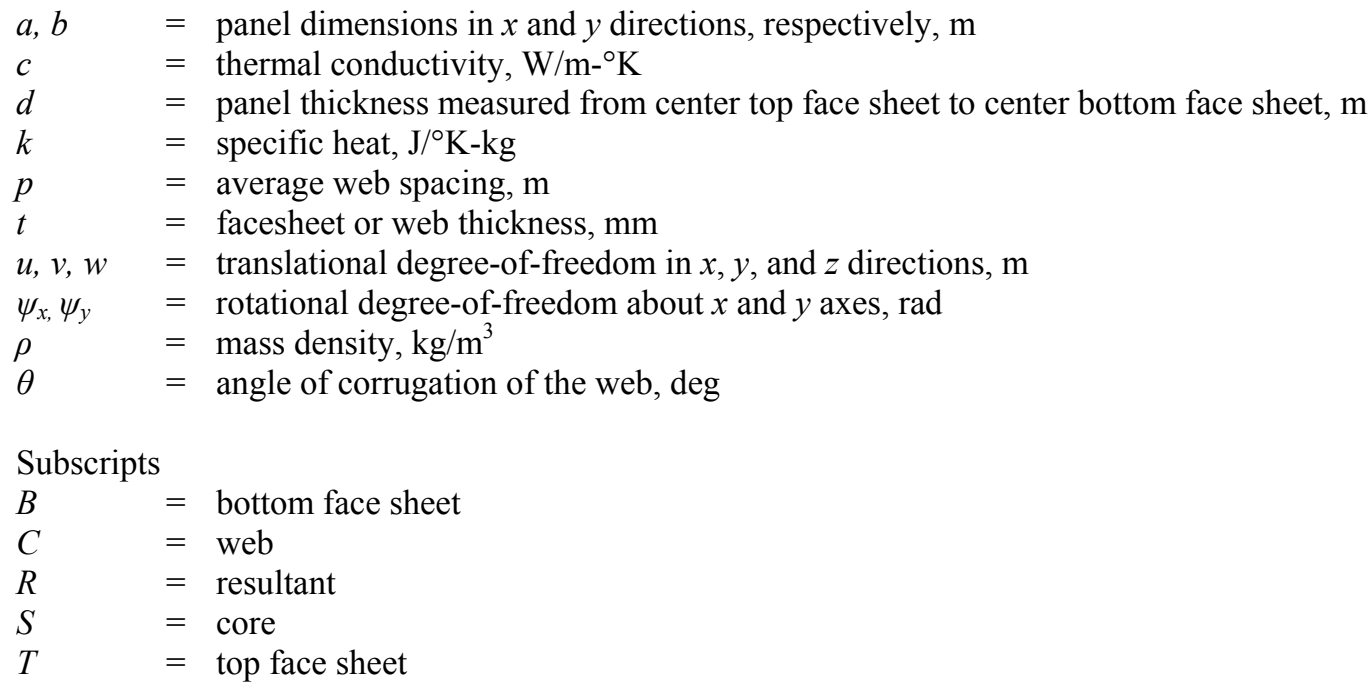

\footnotetext{
${ }^{1}$ Graduate Student, Department of Mechanical and Aerospace Engineering, 231 MAE-A, P.O. Box 116250.

${ }^{2}$ Graduate Student, Department of Mechanical and Aerospace Engineering, 231 MAE-A, P.O. Box 116250.

${ }^{3}$ Senior Structural Engineer, 107 Research Dr., Senior Member AIAA.

${ }^{4}$ Senior Research Engineer, Structural Acoustics Branch, MS 463, Associate Fellow AIAA.
} 


\section{Introduction}

$\mathrm{T}$ he thermal protection system is a critical component of any spacecraft re-entering the atmosphere. When a spacecraft re-enters a planetary atmosphere, it is travelling at hypersonic speeds, resulting in significant aerodynamic heating that can destroy a vehicle without a thermal protection system (TPS). The high temperatures developed over the vehicle may exceed the maximum working temperature or may result in unacceptable structural deformations due to large thermal stress levels. Currently there are two common types of TPS. Capsules, such as those previously used in the Mercury, Gemini, and Apollo programs, currently used in the Soyuz vehicle, and under consideration in future vehicles, typically use an ablator that burns off the vehicle as it absorbs the heat. The Space Shuttle Orbiter uses high temperature resistant ceramic tiles and blankets which act as insulators. Both types of TPS are structurally weak and can easily become damaged, as in the Space Shuttle Columbia accident [1]. An integrated thermal protection system (ITPS) has the potential to not only provide thermal insulation, but also carry structural loads.

Generally, ITPS structures are exposed to combined high-intensity loading environments including thermal, mechanical, aerodynamic, and acoustic loadings. While response analyses and optimization of such structures were considered in the past, the majority of the studies included only a subset of the combined loading environment, e.g., only the thermal environment or only the acoustic environment. Analysis of combined loading conditions, however, is required for more effective structural design. Since the problems of interest are potentially nonlinear, the superposition technique cannot be applied, and therefore an integrated analysis approach is required. Additionally, due to structural complexity of aerospace structures, a finite-element (FE) approach is necessary to achieve the desired modeling fidelity.

The present work offers a procedure utilizing the commercial FE code Abaqus to conduct an analysis of an ITPS structure under a combined loading environment typical of a hypersonic vehicle mission cycle. The procedure is tailored to efficiently handle various loads characterized by significantly different time-scales. Specifically, the slowly-varying heat transfer problem and quasi-static stress analysis under thermal and/or aerodynamic pressure loads are solved using the implicit solvers, while the random response analysis required for rapidly changing acoustic pressure uses an explicit solver. While the procedure is ultimately applied to a large ITPS structure, initial studies aimed at validating the procedure and exploring the effect of individual loadings are conducted on a reducedsize model to keep the computational burden contained. Application of this procedure in future studies is expected to lead to a greater understanding of the thermal-acoustic response of ITPS structures, and ultimately to more robust, weight efficient designs.

\section{ITPS Finite Element Modeling}

The metallic ITPS structure considered in the present studies is presented in Figure 1. A thermal insulation core material present in the entire volume between the top face sheets (TFS), the bottom face sheets (BFS), and the webs is not depicted for clarity. The areal dimensions of the panel are $0.817 \mathrm{~m} \mathrm{x} 0.817 \mathrm{~m}(x \times y)$.

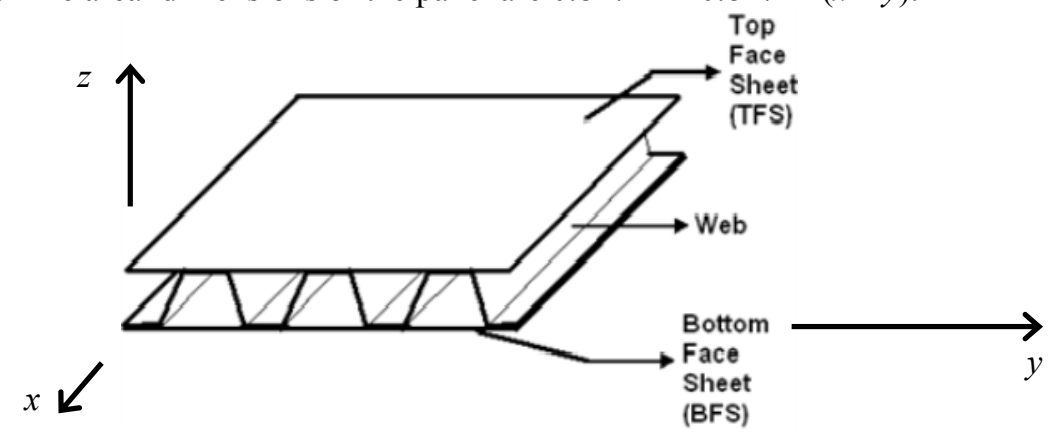

Figure 1. ITPS Panel (shown without thermal insulation core).

The above panel concept was studied extensively in the past for structural optimization under thermal loading only [2-5]. The geometric parameters explored in the optimization studies for fixed areal panel dimensions are illustrated in Figure 2. In the present studies, the following dimensions are adopted: $d=0.090 \mathrm{~m}, 2 \mathrm{p}=0.102 \mathrm{~m}, \mathrm{t}_{\mathrm{C}}=$ $1.20 \mathrm{~mm}, \mathrm{t}_{\mathrm{T}}=1.20 \mathrm{~mm}, \mathrm{t}_{\mathrm{B}}=3.91 \mathrm{~mm}, \theta=89.8^{\circ}$. 


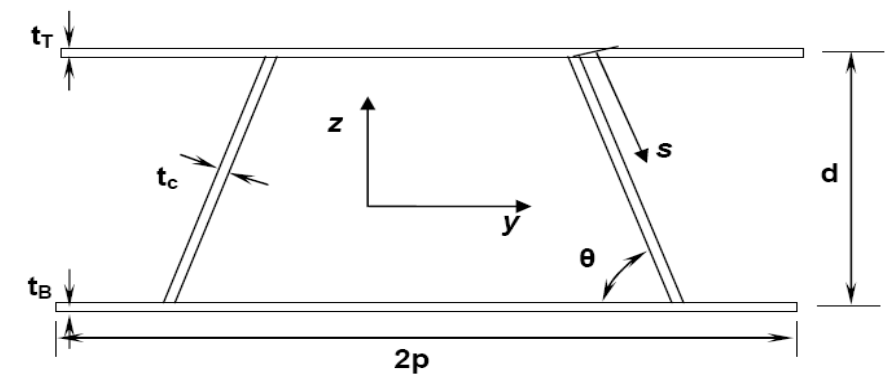

Figure 2. Unit-cell sizing of the corrugated-core sandwich structure.

\section{A. Heat Transfer Model}

A one-dimensional (1-D) heat transfer analysis was conducted using Abaqus 3-node DC1D3 diffusive heat transfer link elements [6]. The total of 30 elements were used through the thickness. They were oriented along the thickness of the panel such that the BFS and TFS were discretized with five equal length elements each $(0.782 \mathrm{~mm}$ and $0.240 \mathrm{~mm}$, respectively), and the core material between the face sheets was discretized with 20 equal length elements ( $4.37 \mathrm{~mm}$ each). This simplified approach was earlier justified by comparing the results obtained from 1-D and 2-D heat transfer analyses by Bapanapalli [5]. The panel's core and webs were modeled as a thermally homogenous resultant material with its resultant conductivity and specific heat being determined through the rule of mixtures as [4-5]

$$
c_{R}=\frac{\rho_{C} c_{C} t_{C}+\rho_{S} c_{S}\left(p \sin \theta-t_{C}\right)}{\rho_{C} t_{C}+\rho_{S}\left(p \sin \theta-t_{C}\right)}
$$

and

$$
\mathrm{k}_{\mathrm{R}}=\frac{\mathrm{k}_{\mathrm{C}} \mathrm{d}_{\mathrm{C}}+\mathrm{k}_{\mathrm{S}}\left(\mathrm{p} \sin \theta-\mathrm{t}_{\mathrm{C}}\right)}{\mathrm{p} \sin \theta} .
$$

Thermal conductivity and specific heat for the titanium alloy TFS and BFS are presented in Figure 3. The same quantities for the Saffil ${ }^{\circledR}$ insulating material are presented in Figure 4.

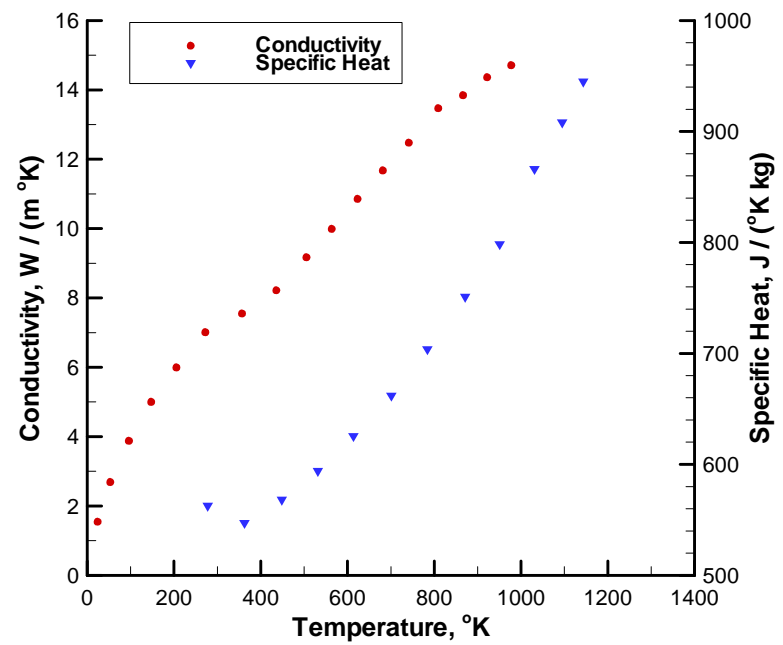

Figure 3. Titanium alloy thermal conductivity and specific heat.

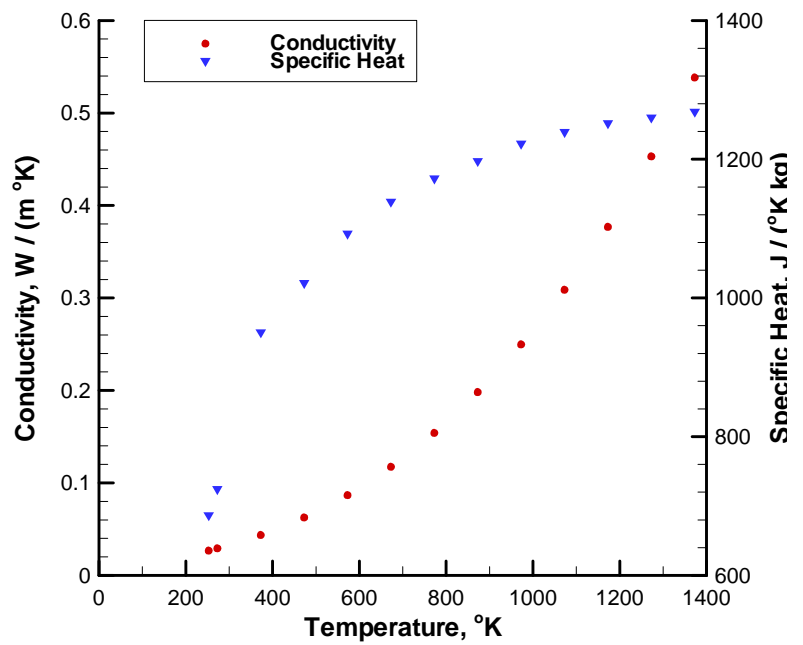

Figure 4. Saffil ${ }^{\circledR}$ thermal conductivity and specific heat.

The lower surface of the BFS was modeled as perfectly insulated and was prescribed an emissivity of zero. The upper surface of the TFS was modeled with radiative and convective boundary conditions (BCs). The emissivity was set to 0.8 and the heat transfer rate per unit area (so-called film coefficient) was set to $5.0 \mathrm{~W} /\left(\mathrm{m}^{2} \mathrm{~K}\right.$ ). 


\section{B. Mechanical Models - Full Panel and Strip Panel}

The full panel model is presented in Figure 5. The model of the 3-D built-up structure was constructed using Abaqus S4R reduced-integration thin-shell elements with three translational and three rotational degrees-of-freedom (DoFs) per node [6]. Mesh convergence was ascertained by reducing the dimensions of the element by half and repeatedly solving a stress-free eigenvalue problem until the difference in the fundamental frequency was reduced to below $2 \%$. This procedure resulted in the element edge size between 8.46 and $8.56 \mathrm{~mm}$ in the direction across the webs (the $y$-direction in Figure 1) and $9.24 \mathrm{~mm}$ along the webs ( $x$-direction). Note that a slightly non-uniform element size on the TFS and BFS in the $y$-direction was dictated by the fact that the webs are not perpendicular to the face sheets. The webs themselves were discretized with elements sized $9.00 \times 9.24 \mathrm{~mm}$, where the first dimension is measured along the $s$-direction in Figure 2. The mechanical BCs of the panel are also presented in Figure 5. The mechanical material properties of the core were considered negligible when compared to the titanium alloy face sheets and webs. Likewise, the core mass inertia effects were considered insignificant. Consequently, the core material was not modeled in the thermal stress and dynamic response analyses.

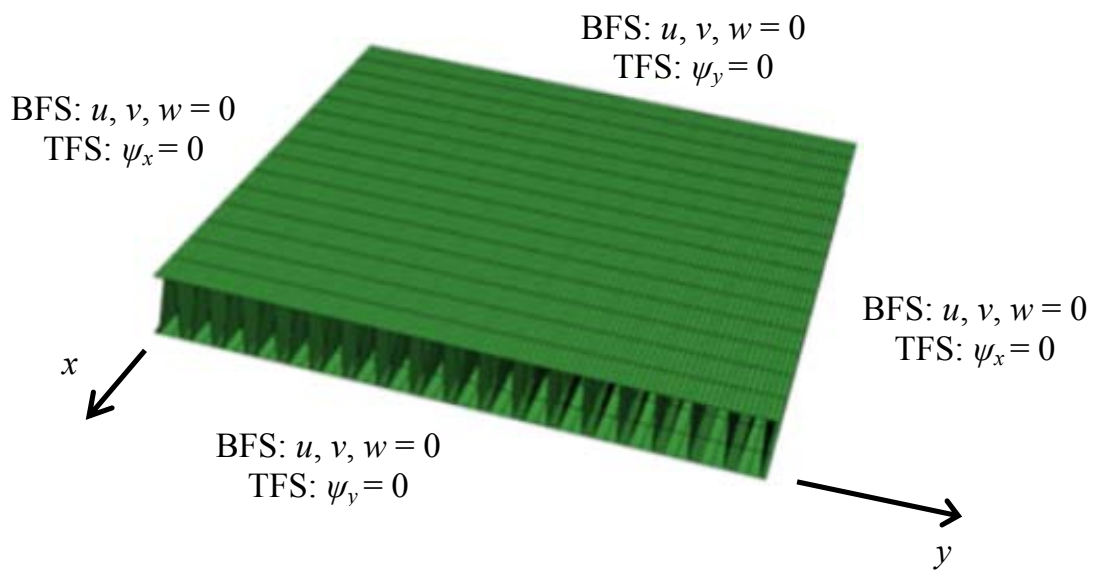

Figure 5. FE model of the full ITPS panel.

Temperature-dependent Young's modulus and thermal expansion coefficient for the titanium alloy are presented in Figure 6. A temperature independent Poisson's ratio of 0.29 and the mass density of $4429 \mathrm{~kg} / \mathrm{m}^{3}$ were specified. In the dynamic response analyses, a $2 \%$ critical modal damping corresponding to the fundamental frequency obtained under the pre-stressed condition (combined thermal and static pressure loadings) of interest was prescribed.

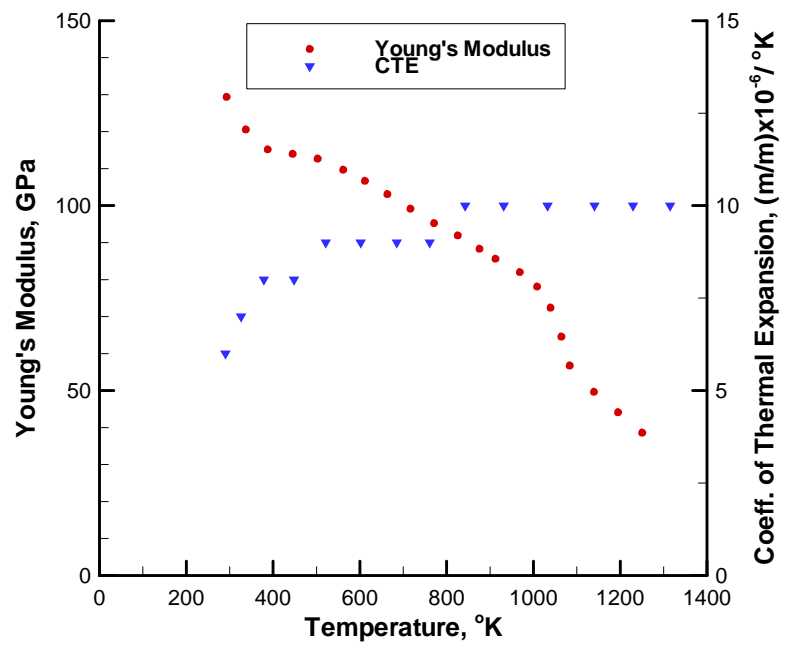

Figure 6. Titanium alloy temperature-dependent material properties.

The size of the full panel FE model as detailed above approached 200,000 DoFs and therefore was considered impractical for conducting initial studies aimed at validating the analysis procedure and exploring the effects of individual loadings. Consequently, a reduced-size model was developed by cutting the $x$ dimension of the panel 
along the web direction to $0.102 \mathrm{~m}$, i.e. to one-eight of its original width. This model is subsequently referred to as a strip panel model. The size of the strip panel model was reduced to approximately 24,000 DoFs. Figure 7 presents the FE strip panel model discretized with the same size S4R elements as outlined for the full panel model.

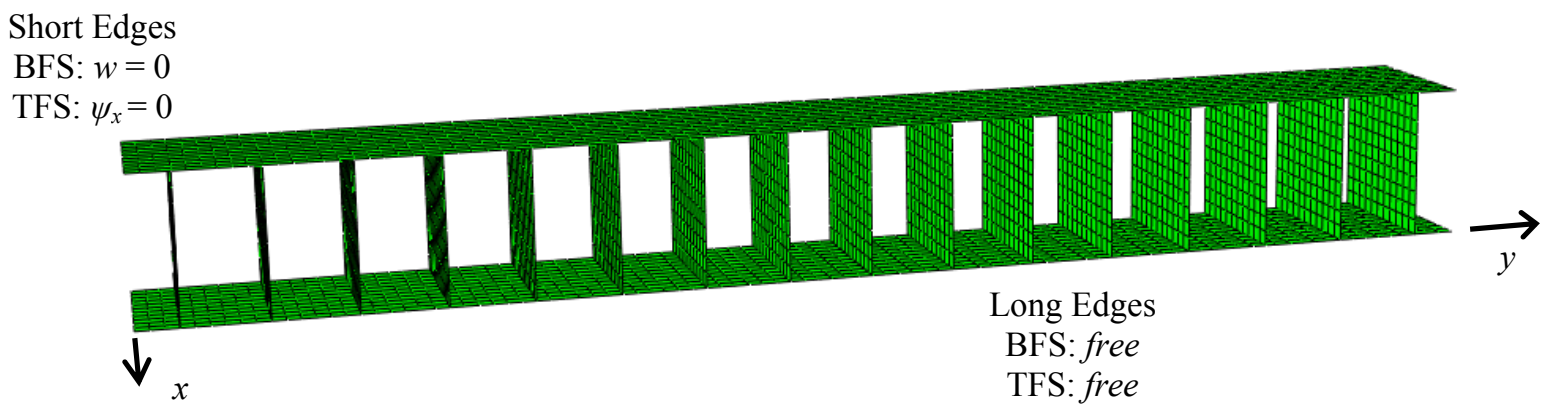

Figure 7. Strip plate FE model.

The material properties of the strip panel model remained unchanged relative to the full plate model. The mechanical BCs, however, were modified. On the longer (span-wise) edges of the panel, all constraints were released. The shorter edges remained supported such that the TFS edges were restricted against the rotation about their respective longitudinal directions and the BFS edges were restricted against the transverse displacement. For the purpose of the dynamic response analysis, the modal critical damping value of $2 \%$ corresponding to the fundamental frequency of the pre-stressed (combined thermal and static pressure loading) structure was maintained. The strip panel fundamental frequency itself differed from that of the full panel.

\section{Analysis Procedure}

\section{A. Loading Definition}

The prescribed heat flux profile and static aerodynamic pressure profile, representative of a typical atmospheric re-entry, are presented in Figure 8. The ambient air temperature was approximated as $243^{\circ} \mathrm{K}$ until $1630 \mathrm{~s}$ into the re-entry and as $273{ }^{\circ} \mathrm{K}$ for the remainder of the re-entry. The acoustic loading spectrum was modeled as truncated white noise with a cut-off frequency of $512 \mathrm{~Hz}$. Two acoustic loading levels were considered - an overall sound pressure level (OASPL) of $130 \mathrm{~dB}$ (re: $20 \mu \mathrm{Pa}$ ) for the strip panel, and OASPLs of $130 \mathrm{~dB}$ and $170 \mathrm{~dB}$ for the full panel. The acoustic pressure time history used in the explicit analysis was generated assuming a random phase distribution. The power spectral density (PSD) of the $130 \mathrm{~dB}$ acoustic loading is presented in Figure 9.

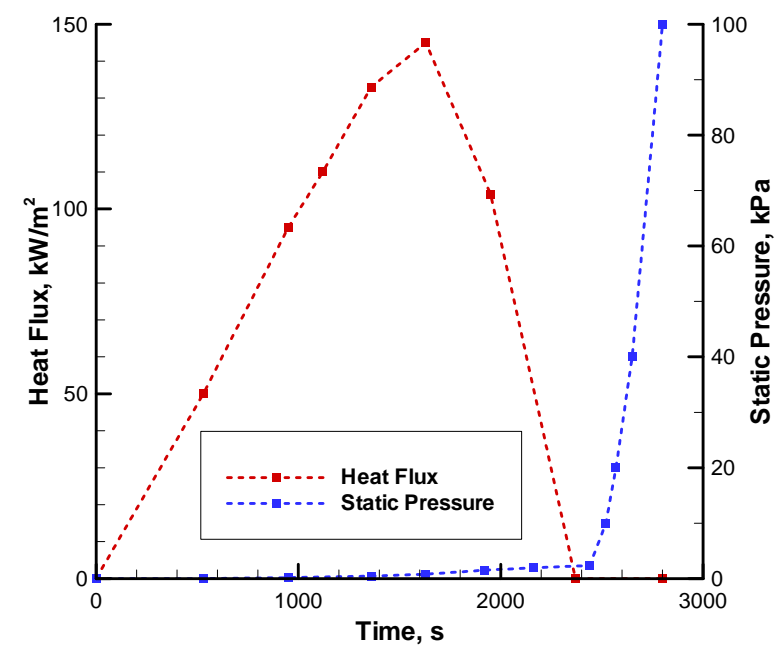

Figure 8. Heat flux profile and static aerodynamic pressure profile during re-entry.

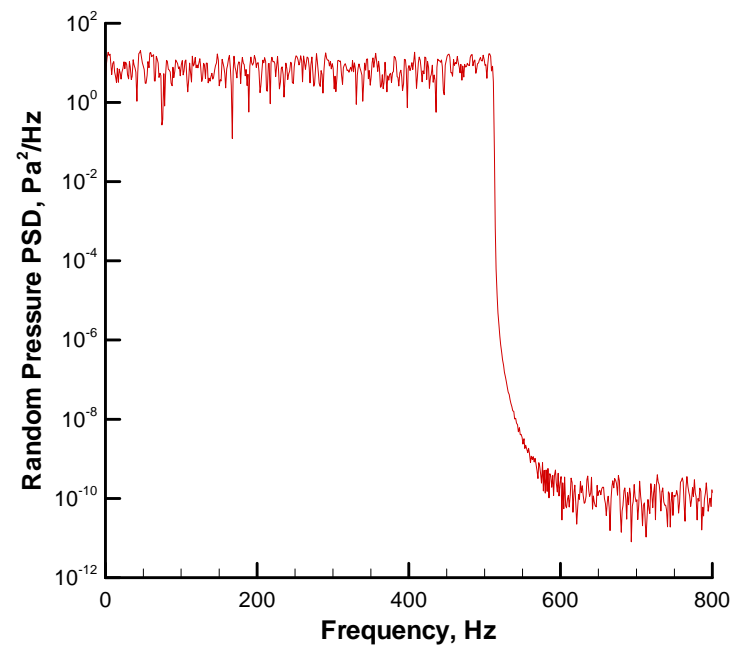

Figure 9. Random pressure PSD at OASPL of 130dB and cut-off frequency of $512 \mathrm{~Hz}$. 


\section{B. Solution Sequence}

A block diagram of the solution procedure applicable to both full and strip panels is presented in Figure 10. The pre-processing steps are depicted in the upper left corner of the diagram and comprise of the heat transfer and structural FE model development, as described in sections II.A and II.B, respectively. The pre-processing step also includes preparation of the loading definitions per section III.A. The loadings are depicted on the right-hand-side of the diagram in the sage-shaded frames.

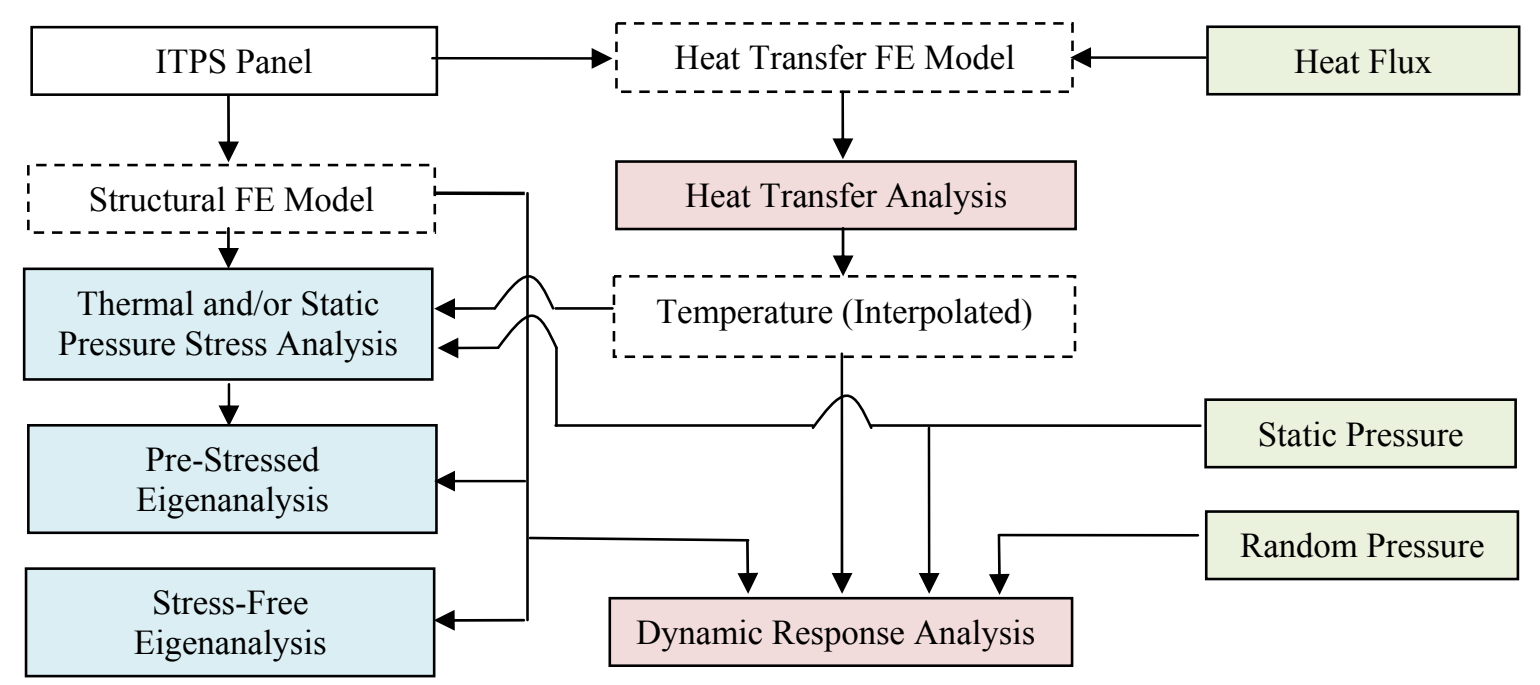

Figure 10. Combined loading solution procedure.

The core of the analysis is comprised of two consecutive Abaqus runs depicted in the center of the diagram in the red-shaded frames. The first step is an Abaqus Standard heat transfer analysis, where the heat transfer model is used to produce a temperature distribution through-the-panel-thickness over the duration of the re-entry profile. Since the through-the-panel-thickness node locations in the heat transfer and structural FE models do not necessarily coincide, the temperature distribution computed from the heat transfer analysis may need to be interpolated before it is applied to the structural FE model, as depicted by the dashed frame below the heat transfer analysis frame.

The second step in the procedure is an Abaqus Explicit dynamic response analysis, where the elevated temperature distribution, static aerodynamic pressure, and random pressure fluctuations are applied simultaneously, as depicted at the bottom of the diagram. This analysis is conducted on a much smaller time scale than the heat transfer analysis as the random pressure fluctuations contain high-frequency components. Typically, the duration of the random response analysis needed for a converged solution statistics is measured in tens of seconds [7-8], i.e. approximately two orders of magnitude less than the duration of a typical re-entry. Effectively, for the purpose of the Abaqus Explicit dynamic response analysis, the temperature distribution and the static aerodynamic pressure are considered constant. Dynamic response analysis results may include displacement, strain, and/or stress time histories.

Finally, apart from the two core analysis steps depicted in Figure 10, the bottom left-hand-side of the diagram includes three additional analyses depicted with the blue-shaded frames. They are considered supportive in nature, and are not mandatory in the procedure execution. The results obtained from these optional steps are often valuable in the interpretation of results. The first optional step, following the required heat transfer analysis, is a static stress analysis performed in Abaqus Standard. This analysis includes thermal and/or static pressure loadings. Generally, it may not be intuitive which of many temperature distributions obtained at different heat transfer analysis time steps cause the most severe stress levels. This is complicated by the fact that static aerodynamic pressure may be present, and that the location of the highest stress is likely to change with changing temperature distribution. Solution of the static stress problem can therefore be helpful in determining whether, for example, the largest thermal stress levels occur when either the TFS or BFS reaches its maximum temperature, when the difference between them is a maximum, or when there is some other distribution. If the solution of the static stress analysis yields prohibitively high stress levels, redesign of the structure or alteration of the boundary conditions may be required. 
One or more optional eigenanalyses, performed on the stress-free and/or statically pre-stressed structure, can also provide valuable information about the dynamic characteristics of the system. In general, compressive thermal stresses can soften the structure in the flat configuration or stiffen the structure in a thermally deflected configuration which in turn can promote a spring-hardening type of a geometric nonlinearity. These softening or hardening characteristics due to the static loads are reflected in decreasing or increasing natural frequencies of the pre-stressed structure when compared to the natural frequencies of the stress-free structure.

\section{Results}

The modeling approach outlined in section II and the solution procedure presented in section III were applied to the strip panel and the full panel models. The heat transfer analysis used to obtain the through-the-panel-thickness temperature distribution is a common prerequisite to both models under consideration. Therefore, the heat transfer results are first presented. The structural analysis results for the strip panel model are presented in section IV.A. Since the strip panel model can be solved in a more time efficient fashion than the full panel model, the effect of the three individual loading components are investigated, namely, thermal, static pressure, and acoustic pressure. The results from a more computationally taxing analysis of the full panel model under combined loadings follows in section IV.B to demonstrate the ability of the procedure to handle large, more realistic ITPS structures.

The 1-D heat transfer problem was solved for the full duration of the re-entry with the heat flux profile presented in Figure 8. Figure 11 shows three snapshots of the through-the-panel-thickness temperature distribution of the ITPS panel when (i) the temperature at the BFS is a maximum, (ii) the temperature at the TFS is a maximum, and (iii) the temperature difference between the TFS and BFS is a maximum. The latter temperature distribution, exhibiting a temperature differential of $779^{\circ} \mathrm{K}$, was selected for all subsequent analyses involving thermal loading because previous optimizations studies showed this case to yield the highest stress levels [5]. It is interesting to note in Figure 11 that nearly isothermal conditions of the BFS and the TFS (the latter is significantly thinner than the BFS) are present for all three temperature snapshots.

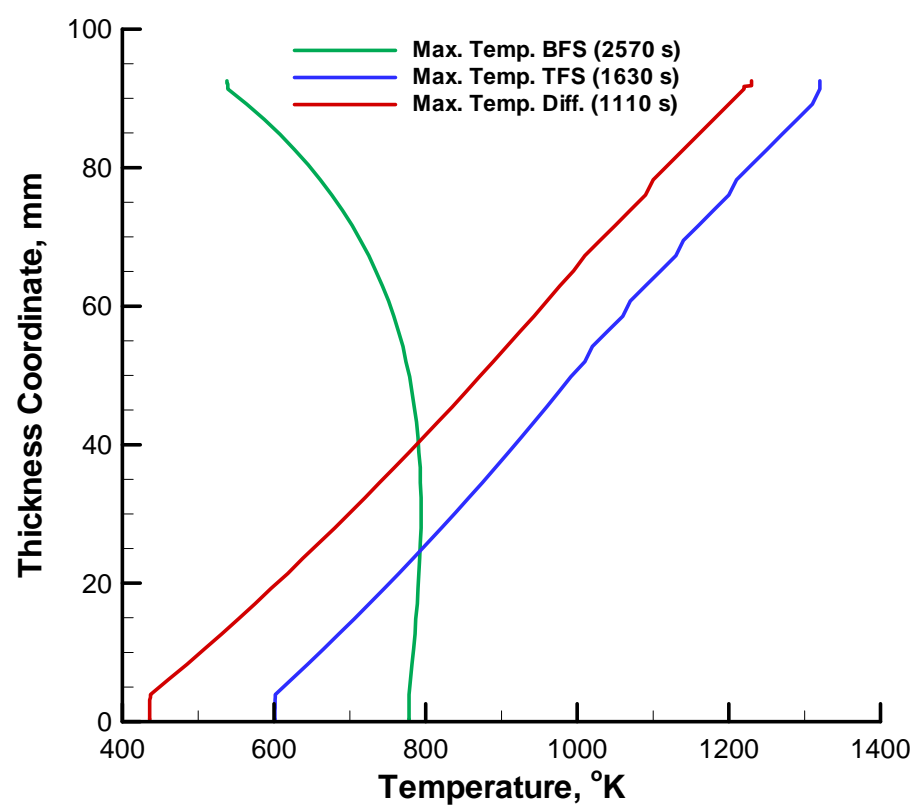

Figure 11. Various temperature distributions during re-entry.

\section{A. Strip Panel Model}

The von Mises stress and displacements were first computed using a nonlinear static analysis, as indicated in the top blue-shaded frame of Figure 10. Three distinct static loading scenarios were considered to gain insight into their individual and combined effect on the structure, i.e., thermal loading only, static pressure only, and combined thermal loading and static pressure. The prescribed through-the-panel-thickness temperature distribution shown by the red curve in Figure 11 corresponded to the re-entry time of 1110 seconds. The corresponding static aerodynamic pressure has a value of $356 \mathrm{~Pa}$, as presented in Figure 8. 
The von Mises stress and transverse displacement fields due to the thermal loading only are presented in Figure 12 and Figure 13, respectively. The maximum stress of $72.1 \mathrm{MPa}$ is located in the two webs closest to the ends of the strip panel, at the edge elements attached to the BFS. The maximum transverse deflection of $5.81 \mathrm{~mm}$ is located at the half-span location of the TFS. Since the TFS is heated to a much higher temperature than the BFS, the upward TFS deflection is expected. The von Mises stress and transverse displacement fields due to the static pressure only are presented in Figure 14 and Figure 15, respectively. The maximum stress of $9.02 \mathrm{MPa}$ is located in the webs second closest to the end of the strip panel, where the TFS intersects the web. The maximum transverse deflection of $-0.63 \mathrm{~mm}$ is located at the half-span location of the TFS. Since the pressure loading acts downwards, the panel deflection is opposite to that caused by the thermal loading. The von Mises stress and transverse displacement fields due to the combined thermal and pressure loading are presented in Figure 16 and Figure 17, respectively. The maximum stress of $79.7 \mathrm{MPa}$ coincides with the location of the maximum stress levels computed from the thermal load only case. The maximum transverse deflection of $4.79 \mathrm{~mm}$ is again located at the half-span location of the TFS.

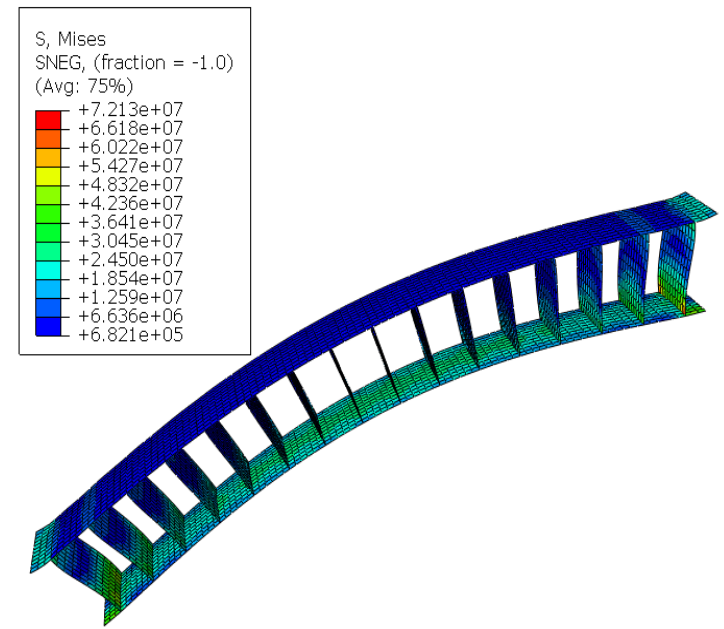

Figure 12. Von Mises stress due to the thermal load, Pa.

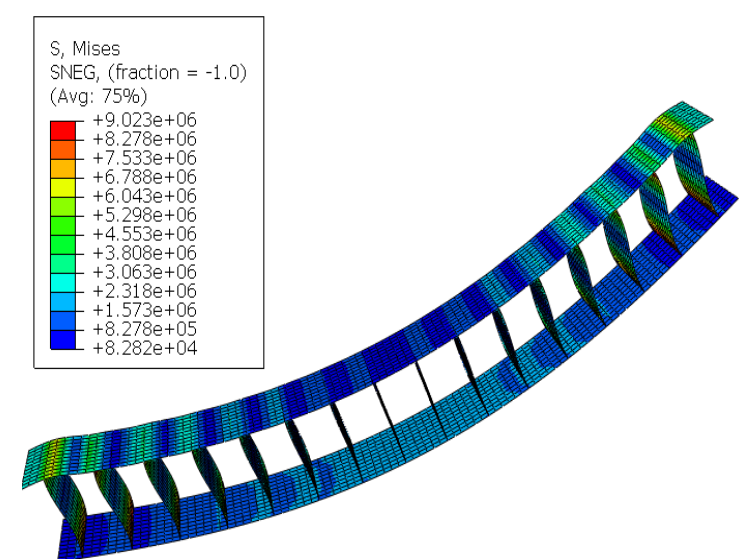

Figure 14. Von Mises stress due to the static pressure load, $\mathbf{P a}$.

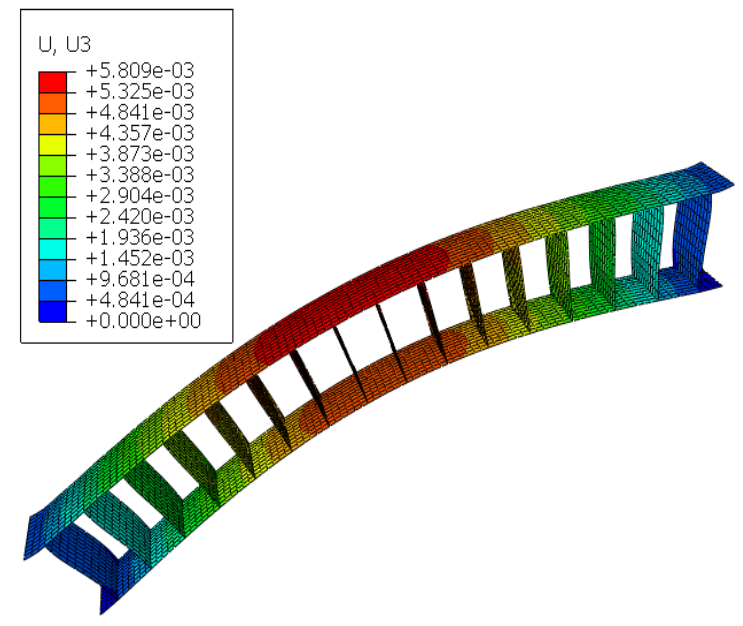

Figure 13. Transverse displacement field for the thermal load, $\mathbf{m}$.

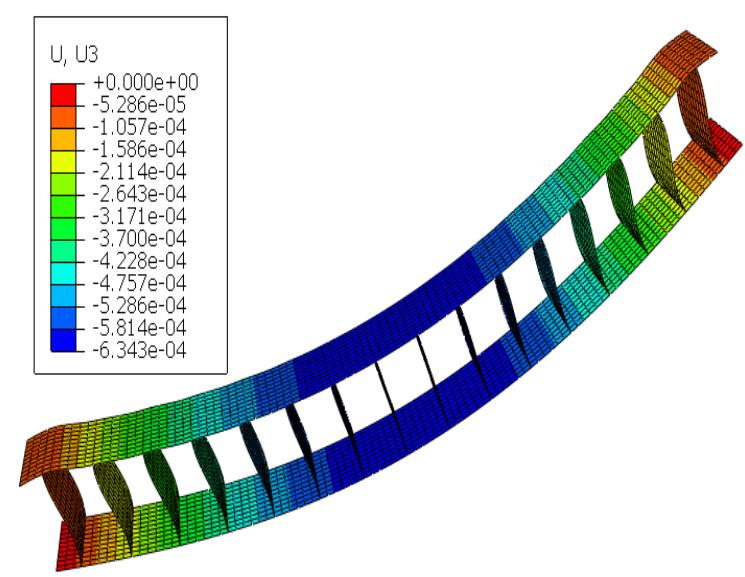

Figure 15. Transverse displacement field due to the static pressure load, $\mathbf{m}$. 


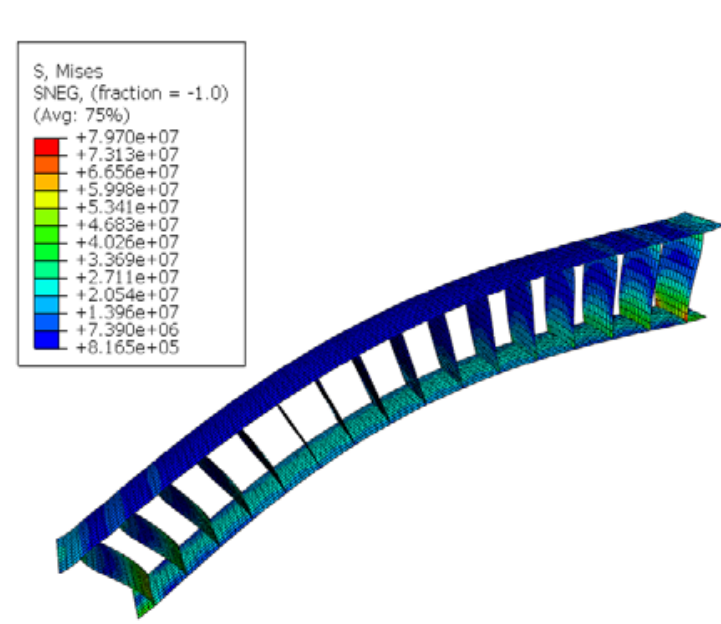

Figure 16. Von Mises stress due to the combined thermal and static pressure loading, Pa.

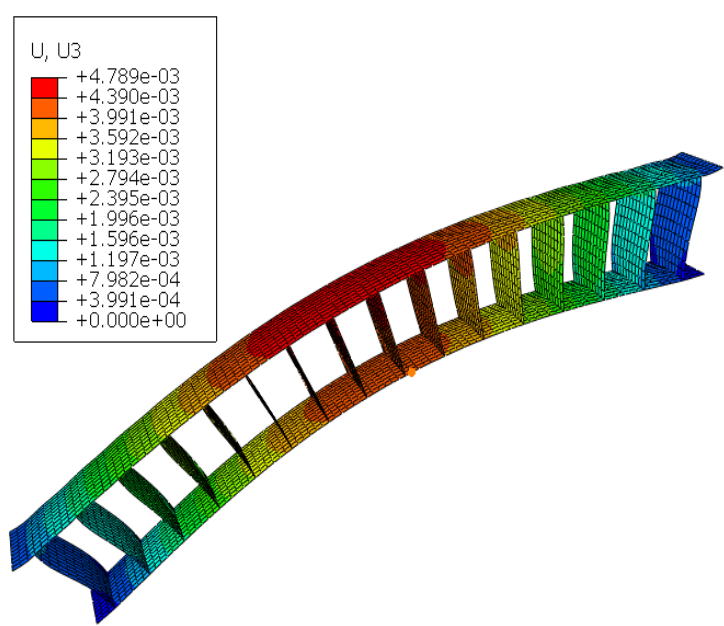

Figure 17. Transverse displacement field due to the combined thermal and static pressure loading, $\mathbf{m}$.

Table 1 summarizes the results of the static analyses. For all three static cases analyzed, the maximum von Mises stress is well under the Titanium-4V-6Al yield stress of $1000 \mathrm{MPa}$. The effect of the applied static pressure only is small compared to that of the thermal loading only. The static pressure applied in addition to the thermal loading results in a decreased maximum deflection when compared to the thermal loading only case. Despite the reduced transverse deflection, however, the combined load results in a higher maximum stress level when compared to the thermal loading only case. Finally, it is also worthwhile to note that the displacement results presented in Table 1 demonstrate that the structure responds nonlinearly to the loading levels being applied. Note that the summation of the collocated maximum transverse deflection values from the two individual loading cases differs from the combined loading case by $8 \%$. The static deflection calculated in these analyses is dictated by the stiffness, which itself was previously found to converge, via eigenanalyses, to within $2 \%$. Therefore, the $8 \%$ difference is in excess of the uncertainty of the static analyses.

Table 1. Results of the nonlinear static analyses of the strip panel.

\begin{tabular}{ccc}
\hline \hline Loading Condition & $\begin{array}{c}\text { Maximum von Mises Stress, } \\
\text { MPa }\end{array}$ & $\begin{array}{c}\text { Maximum Transverse Displacement, } \\
\text { mm }\end{array}$ \\
\hline Thermal Only & 72.1 & 5.81 \\
\hline Static Pressure Only & 9.02 & -0.63 \\
\hline Combined Thermal and Pressure & 79.7 & 4.79 \\
\hline \hline
\end{tabular}

Following the series of static analyses, four eigenanalyses were performed. Apart from the stress-free eigenanalysis, three pre-stressed eigenanalyses corresponding to the three loading conditions outlined in Table 1 were also conducted. The frequencies associated with the first three symmetric modes are presented in Table 2 for the stress-free and the three pre-stressed cases. It is seen that the thermal stresses result in a softening of the panel characteristics relative to the stress-free condition, as indicated by the reduced natural frequencies. The static pressure only pre-stressed eigenproblem yielded nearly identical results as the stress-free eigenproblem. The prestressed structure for the combined loading case yielded nearly identical results as the thermal stress only case. The results obtained from the nonlinear static analyses are consistent this observation, i.e., the effect of the static pressure loading is minor when compared to the thermal loading.

Table 2. Natural frequencies of the stress free and pre-stressed strip panel.

\begin{tabular}{ccccc}
\hline \hline Mode \# & Stress-Free & $\begin{array}{c}\text { Thermally } \\
\text { Pre-Stressed }\end{array}$ & $\begin{array}{c}\text { Frequency, Hz } \\
\text { Pre-Stressed }\end{array}$ & $\begin{array}{c}\text { Combined Thermal and } \\
\text { Pressure Pre-Stressed }\end{array}$ \\
\hline 1 & 23.64 & 18.72 & 23.64 & 18.72 \\
\hline 2 & 111.6 & 99.74 & 111.6 & 99.73 \\
\hline 3 & 275.4 & 255.3 & 275.4 & 255.2 \\
\hline \hline
\end{tabular}


Next, a nonlinear dynamic response analysis under a $130 \mathrm{~dB}$ acoustic pressure loading combined with the thermal and static pressure loads was performed. The panel was assumed to be initially at rest. The combined acoustic and static pressure loading was applied from the beginning of the simulation, while the temperature distribution was linearly ramped up from zero to the target distribution over a period of $0.5 \mathrm{~s}$ to minimize the thermally induced transient dynamic response. Figure 18 presents the transverse displacement response at the TFS center node. Figure 19 presents the stress response at the bottom edge element of the first web in the in-plane direction aligned with the ITPS panel depth (i.e., along the $s$-coordinate in Figure 2). This is the same element where the static stress response reaches the maximum value. The initial $0.5 \mathrm{~s}$ of the simulation, during which the thermal loading is linearly ramped up, is removed from Figure 18 and Figure 19 and only the part of the response under the time-invariant temperature distribution is presented. It is interesting to note that the initial portion of the remaining displacement and stress responses contain frequency components noticeably higher than the latter portion. This is best observed in the frequency domain response of the each portion (initial and latter), not shown for brevity. There, it is seen that the participation of high-frequency modes is greater in the stress response than in the displacement response. Further, because of the way in which the damping is specified, the higher-frequency modes are less damped than the lower-frequency modes. Therefore, the high-frequency behavior in the stress response persists for a longer duration $(\sim 0.9 \mathrm{~s})$ than in the displacement response $(\sim 0.6 \mathrm{~s})$.

The dashed horizontal lines in Figure 18 and Figure 19 indicate the static displacement and stress, respectively, under the combined thermal and static aerodynamic pressure loading. The transverse displacement in Figure 18 is seen to oscillate around a deflection of $4.79 \mathrm{~mm}$, consistent with the value from Table 1. It is also observed that the dynamic stress response oscillates around $68.0 \mathrm{MPa}$, i.e., the value obtained from the static analysis.

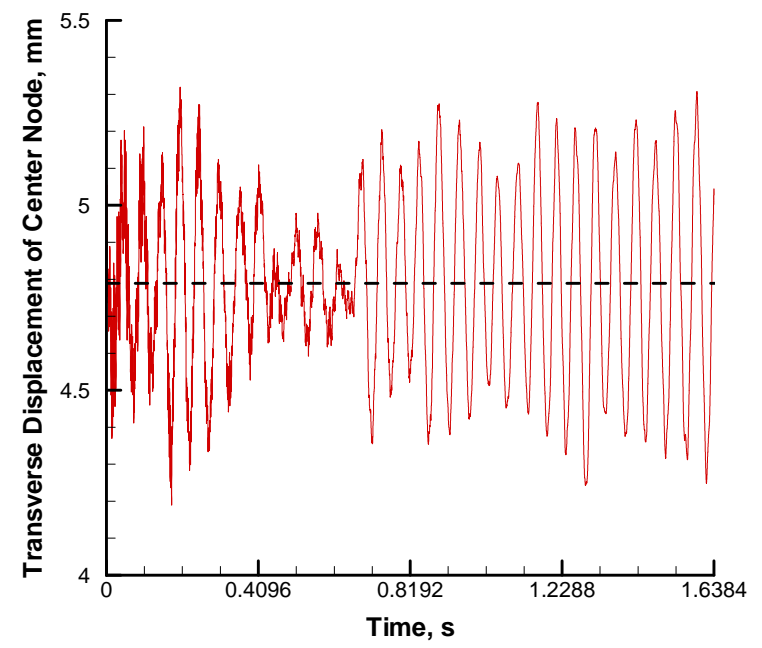

Figure 18. Transverse displacement time response of the TFS center node under combined loading condition.

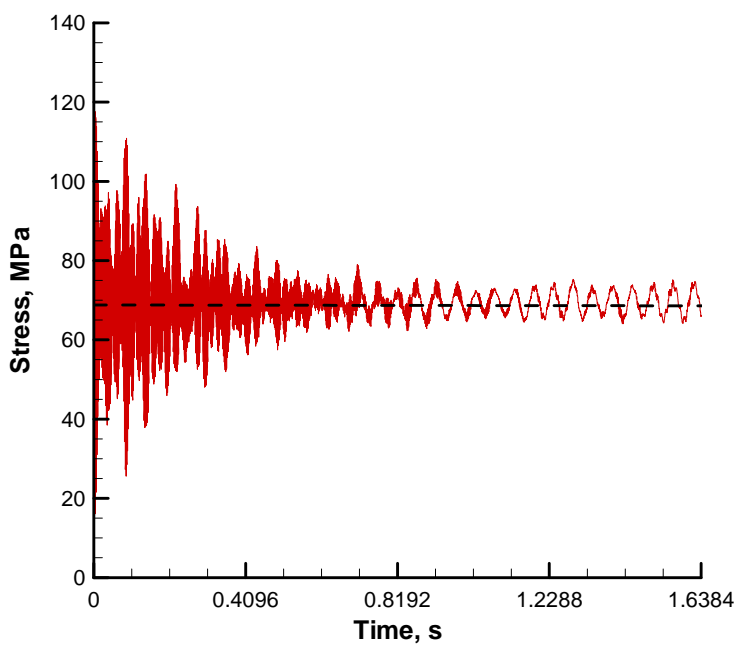

Figure 19. Stress time response in the web direction on an outer web elements exhibiting maximum stress in the thickness direction.

To further investigate the effects of individual loadings in the nonlinear dynamic response regime, four center node transverse displacement PSDs at the TFS are presented in Figure 20. The results include the following loading conditions: (i) random pressure loading only $(130 \mathrm{~dB}$ ), (ii) random pressure loading and static aerodynamic pressure $(130 \mathrm{~dB}+\mathrm{P})$, (iii) random pressure loading and thermal loading $(130 \mathrm{~dB}+\Delta \mathrm{T})$, and (iv) combined random pressure, thermal and static pressure loadings $(130 \mathrm{~dB}+\Delta \mathrm{T}+\mathrm{P})$. The zero-frequency response of any of the statically preloaded conditions (P and/or $\Delta \mathrm{T}$ ) is greater than that of the first resonant peak (roughly $10^{-8} \mathrm{~m}^{2} / \mathrm{Hz}$ ). Although difficult to see in the figure, the zero frequency responses of the $(130 \mathrm{~dB}+\Delta \mathrm{T})$ and $(130 \mathrm{~dB}+\Delta \mathrm{T}+\mathrm{P})$ loadings exceed $10^{-5} \mathrm{~m}^{2} / \mathrm{Hz}$, while the $(130 \mathrm{~dB}+\mathrm{P})$ load case is approximately $10^{-7} \mathrm{~m}^{2} / \mathrm{Hz}$. In contrast, the zero-frequency response of the $130 \mathrm{~dB}$ case is below $10^{-10} \mathrm{~m}^{2} / \mathrm{Hz}$.

The blue vertical dashed lines in Figure 20 indicate the first three natural frequencies obtained from the stressfree eigenanalysis while the black vertical dashed lines mark the first three natural frequencies obtained from the pre-stressed eigenanalysis $(\Delta \mathrm{T}+\mathrm{P})$. Note that only response components with span-wise symmetric transverse displacements, consistent with the symmetry of the structure and the loadings, were excited. The results of the 
random response analysis are also consistent with the eigenanalyses (see Table 2). Specifically, resonant frequencies for load cases (i) and (ii) closely match natural frequencies from the stress-free and static pressure only (P) pre-stressed conditions. Likewise, resonant frequencies from load cases (iii) and (iv) closely match natural frequencies pre-stressed having a thermal loading component $(\Delta T)$. It is seen that for each of the four loading cases presented in Figure 20, the most pronounced dynamic response component is located in the vicinity of the frequency of the lowest symmetric mode. All the peaks present in the excitation bandwidth are relatively well-defined and do not exhibit significant shifting or broadening. This observation further substantiates the conclusion that although the thermal loading introduces deformations sufficiently large to warrant a nonlinear analysis, the random pressure excitation effectively results in a small amplitude perturbation about a large thermally-induced deflection.

It is also noted that the frequency response above approximately $420 \mathrm{~Hz}$ differs in character between those cases having a thermal loading component and those that do not. In those cases having a thermal loading component $(\Delta \mathrm{T})$, several symmetric transverse displacement modes in this high frequency band were identified. In those cases without a thermal loading component $(\Delta \mathrm{T})$, no such modes were identified in the bandwidth. The significant modal density of symmetric transverse components in the high frequency bandwidth not only explains the presence of multiple peaks in the PSD, but also an increased response above the cut-off frequency due to the contribution of additional modes outside the excitation bandwidth.

Finally, note in Figure 20 that while the differences between resonant frequencies and eigenvalues appears smallest (in absolute terms) at the lower frequencies, the relative difference is comparable across the frequency range $(<4 \%)$. It has previously been observed that such differences are due to the two distinct solution techniques being used, i.e., explicit for the dynamic response simulation and implicit for the eigenanalysis. Recall, that only the fundamental frequency was used to assure mesh convergence, as discussed in section II.B.

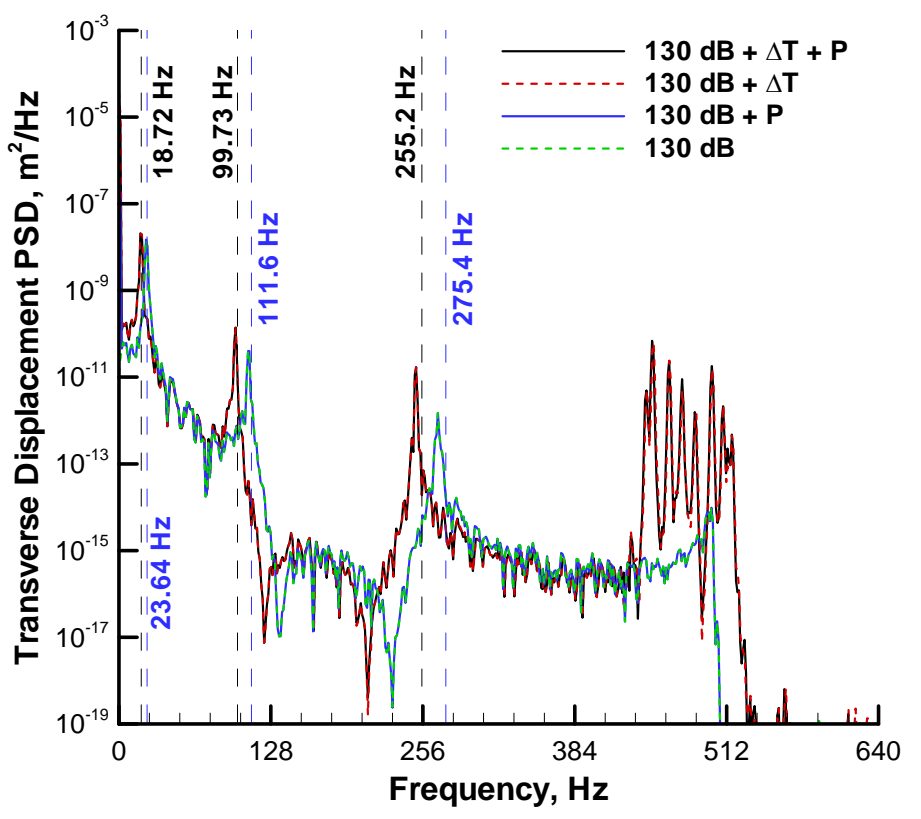

Figure 20. Transverse displacement PSD at the TFS center due to various loadings.

\section{B. Full Panel Model}

A less exhaustive set of analyses of the full panel model was next undertaken to verify that the analysis scheme could be successfully applied to a large structure representative of an ITPS. Per the Figure 10 flowchart, the nonlinear static analyses under the thermal $(\Delta \mathrm{T})$ and/or static aerodynamic pressure loadings $(\mathrm{P})$ were first performed. The same thermal and the static aerodynamic pressure loadings as applied to the strip panel model were applied to the full panel model. Table 3 summarizes the results. It is seen that the deflection at the TFS center due to the static pressure only loading $(\mathrm{P})$ is minimal and the maximum stress level nearly negligible. On the other hand, the thermal loading $(\Delta \mathrm{T})$, with or without the static pressure loading $(\mathrm{P})$ introduces an appreciable deflection of $10.21 \mathrm{~mm}$, i.e. approximately $1 / 9$ the overall panel thickness $d$. It is also observed that the maximum von Mises stress computed under the thermal loading $(\Delta \mathrm{T})$ is high (the results are almost identical for the two cases with and without the static pressure loading) and exceeds the ultimate strength of $1000 \mathrm{MPa}$ for Titanium-4V-6Al. To 
facilitate a more insightful discussion of the stress levels, the full field von Mises stress is presented in Figure 21. Additionally, the transverse displacement field is presented in Figure 22.

Table 3. Results of the static analyses of the full panel.

\begin{tabular}{ccc}
\hline \hline Loading Condition & $\begin{array}{c}\text { Maximum von Mises Stress, } \\
\text { MPa }\end{array}$ & $\begin{array}{c}\text { Maximum Transverse Displacement, } \\
\text { mm }\end{array}$ \\
\hline Thermal Only & 2424 & 10.21 \\
\hline Static Pressure Only & 1.25 & -0.0023 \\
\hline Combined Thermal and Pressure & 2425 & 10.21 \\
\hline \hline
\end{tabular}

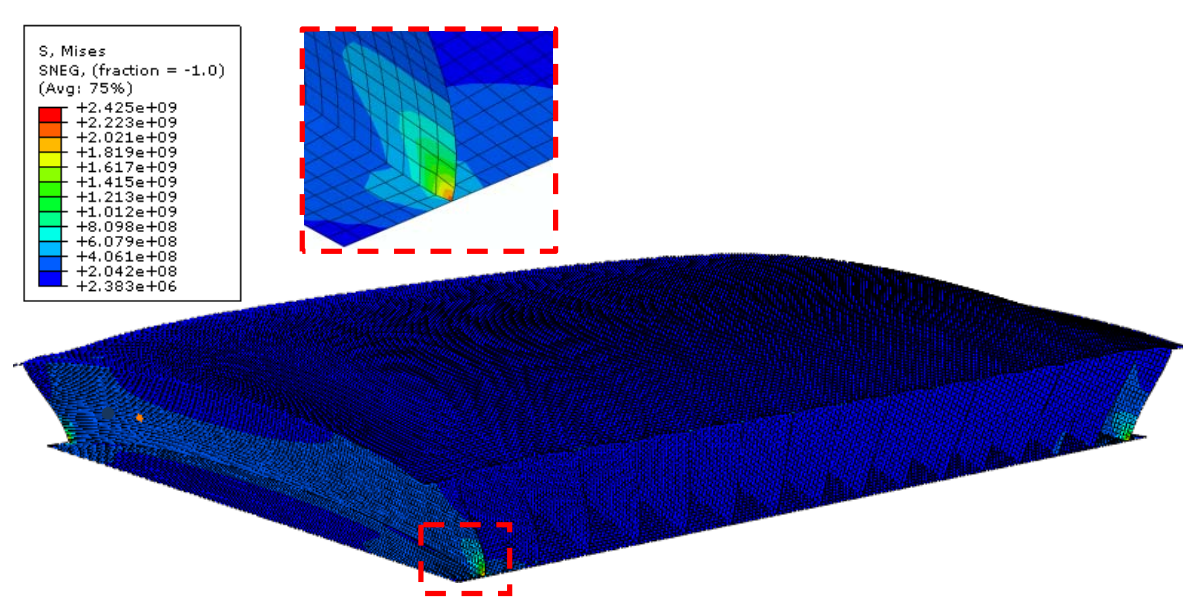

Figure 21. Von Mises stress due to combined thermal and static pressure, Pa.

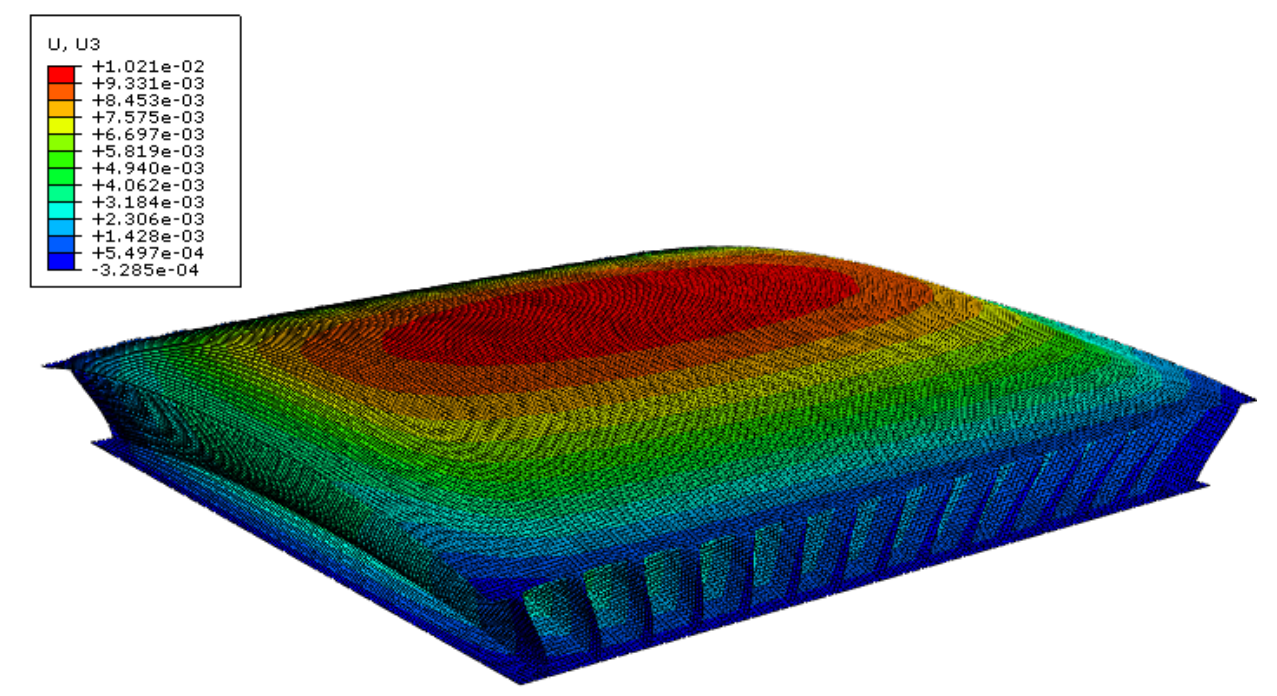

Figure 22. Transverse displacement field for the combined thermal and static pressure loads, $\mathbf{m}$.

It is seen in Figure 21 that the sections of the panel which exhibit von Mises stress levels exceeding $1000 \mathrm{MPa}$ are very localized, whereas the highest von Mises stress levels elsewhere are less than $600 \mathrm{MPa}$, i.e., well within the material allowable. The high stress areas occur in the web sections located near the four corners of the panel where the most outer webs intersect the BTS, as shown in the close-up view. The high stresses induced are attributable to the simplified manner in which the panel BCs were specified, that is, an in-plane immovable condition on the BFS instead of a more realistic compliant condition to allow for thermal expansion. Improved modeling of the BCs, 
consideration of material nonlinearity, and refined mesh discretization in the high stress regions would be required in an actual analysis application. However, these factors do not affect the method development described herein.

The eigenanalysis results of the full panel model indicated it to be stiffer than the strip panel model. For the stress-free and pre-stressed conditions (combined thermal and static pressure loading), the frequencies of the lowest symmetric modes were found to be $435.5 \mathrm{~Hz}$ and $315.6 \mathrm{~Hz}$, respectively. Similar to the strip panel study, a noticeable softening effect resulting from the thermal loading was observed.

Following the eignanalyses, a nonlinear dynamic response simulation under a combined $130 \mathrm{~dB}$ acoustic load, thermal load, and static aerodynamic pressure load was performed. As observed in the case of the strip panel model, the displacements and stresses were found to oscillate around their respective static analysis values (results not shown for brevity). The dynamic part of the response computed for this loading condition was very small. The maximum peak to peak transverse displacement was only about $0.3 \mathrm{~mm}$ about the static displacement of $10.21 \mathrm{~mm}$ (see Table 3).

Subsequently, the combined thermal and static pressure loading was kept at the previous level and the random pressure loading was increased to $170 \mathrm{~dB}$. Such a loading scenario may be found in the vicinity of the propulsion system during powered flight. Under the $170 \mathrm{~dB}$ loading, the transverse oscillations were more pronounced (approximately $2 \mathrm{~mm}$ in amplitude). The displacement PSDs for the $130 \mathrm{~dB}$ and $170 \mathrm{~dB}$ combined loading cases are shown in Figure 23.

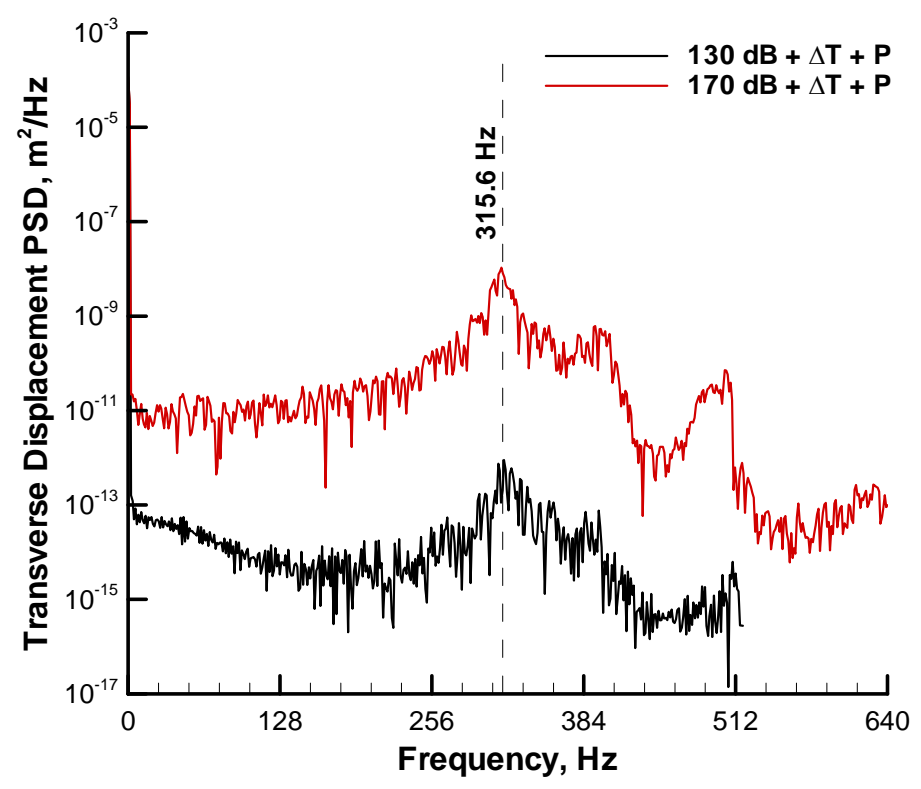

Figure 23. Transverse displacement PSD at the TFS center due to combined loading environment with a varying OASPL.

Figure 23 demonstrates that the most pronounced dynamic response is located in the vicinity of the lowest symmetric mode frequency, depicted with the dashed line. This feature of the response is similar to that of the strip panel model, see Figure 20. While difficult to see in Figure 23, the dominant zero-frequency response, induced by the thermal and aerodynamic pressure loadings $(\Delta \mathrm{T}+\mathrm{P})$, slightly exceeds $10^{-4} \mathrm{~m}^{2} / \mathrm{Hz}$. The dominant dynamic responses are considerably lower than the zero-frequency response (approximately $10^{-12} \mathrm{~m}^{2} / \mathrm{Hz}$ and $10^{-8} \mathrm{~m}^{2} / \mathrm{Hz}$ for the $130 \mathrm{~dB}$ and $170 \mathrm{~dB}$ loadings, respectively). However, peak broadening is substantially more significant than observed in the strip panel model analysis, pointing to a higher degree of nonlinearity which can partially be attributed to the immovable in-plane BCs of the BFS. Additional peaks are seen in the vicinity of $390 \mathrm{~Hz}$ and $500 \mathrm{~Hz}$. The peak around $500 \mathrm{~Hz}$ (truncated at the loading cut-off frequency) becomes more prominent as the acoustic loading is increased from 130 to $170 \mathrm{~dB}$ OASPL. The modal density in the vicinity of both the $390 \mathrm{~Hz}$ and $500 \mathrm{~Hz}$ peaks is high, so it is not possible to correlate the dynamic response with modal participation without the aid of an identification procedure. 


\section{Concluding Remarks}

An analysis procedure to simulate the response of an ITPS structure under combined thermal, aerodynamic, and acoustic loadings was presented. The core of the procedure consists of a heat transfer problem followed by a nonlinear structural dynamic response simulation. Both analyses are carried out using the FE commercial code Abaqus, and both implicit and explicit solutions in the physical DoFs are used.

Two structures were investigated using the procedure. A smaller strip panel model was used to establish confidence in the approach and to investigate individual loading effects. Ultimately, a large ITPS panel was studied to establish applicability of the method to representative ITPS structural analysis and design problems. Both structures exhibited similar response characteristics under the particular set of applied loadings. It was found that the applied thermal loading condition (having the most significant through-the-panel-thickness temperature gradient) contributed most to the overall stress field of both structures and caused large deflections warranting nonlinear structural analysis. High thermal stresses resulted in a softening of both ITPS panels, as indicated by a lower fundamental frequency relative to the stress-free condition. The effect of the static aerodynamic pressure was found to be insignificant when compared to the effect of the thermal loads. Random pressure fluctuations also resulted in relatively small oscillations about the thermally deflected shape. For both structures, the dominant dynamic response occurred at the statically pre-stressed natural frequency of the lowest symmetric mode.

Confidence in the proposed procedure was gained by studying individual loading effects and by comparing results from the core analyses with those from supportive analyses, e.g. static thermal stress and eigenanalyses. Further validation though comparison with experimental data would be valuable. Future work may also focus on incorporating reduced-order simulation [9-10] into the proposed scheme to take advantage of improved computational efficiency afforded by that method. Such an enhancement is required to make application of the procedure to sensitivity studies and optimization problems feasible, leading to the design of hypersonic vehicle structures optimized for combined loading environments. In such applications, improved definition of boundary conditions, consideration of material nonlinearity, and modeling of high stress regions is required.

\section{References}

[1] "Columbia Accident Investigation Board Report," National Aeronautics and Space Administration, Washington, DC, August 2003.

[2] Sharma, A., Sankar, B.V., and Haftka, R.T., "Multi-fidelity analysis of corrugated-core sandwich panels for integrated thermal protection systems," 50th AIAA/ASME/ASCE/AHS/ASC Structures, Structural Dynamics, and Material Conference, AIAA-2009-2201, Palm Springs, CA, 2009.

[3] Gogu, C., Bapanapalli, S.K., Haftka, R.T., and Sankar, B.V., "Comparison of materials for an integrated thermal protection system for spacecraft reentry," Journal of Spacecraft and Rockets, Vol. 46, No. 3, pp. 501513, 2009.

[4] Gogu, C., Haftka, R.T., Bapanapalli, S.K., and Sankar, B.V., "Dimensionality reduction approach for response surface approximations: application to thermal design," AIAA Journal, Vol. 47, No. 7, pp. 1700-1708, 2009.

[5] Bapanapalli, S.K., Martinez, O.M., Gogu, C., Sankar, B.V., and Haftka, R.T., "Analysis and design of corrugated-core sandwitch panels for thermal protection systems of space vehicles," 47th AIAA/ASME/ASCE/AHS/ASC Structures, Structural Dynamics, and Material Conference, AIAA-2006-1942, Newport, RI, 2006.

[6] "ABAQUS User's Manual." Providence, RI,: Dassault Systèmes Simulia Corp., 2009.

[7] Przekop, A., Rizzi, S.A., and Sweitzer, K.A., "An investigation of high-cycle fatigue models for metallic structures exhibiting snap-through response," International Journal of Fatigue, Vol. 30, No. 9, pp. 1579-1598, 2008.

[8] Rizzi, S.A., Behnke, M.N., and Przekop, A., "The effect of non-gaussian random loading on high-cycle fatigue of a thermally post-buckled structure," Structural Dynamics: Recent Advances, Proceedings of the 10th International Conference, The Institute of Sound and Vibration Research, University of Southampton, Southampton, UK, July, 2010.

[9] Przekop, A., Guo, X., and Rizzi, S.A., "Alternative modal basis selection procedures for nonlinear random response simulation," Structural Dynamics: Recent Advances, Proceedings of the 10th International Conference, The Institute of Sound and Vibration Research, University of Southampton, Paper 036, Southampton, UK, 2010.

[10]Rizzi, S.A. and Przekop, A., "Estimation of sonic fatigue by reduced-order finite element based analysis," Structural Dynamics: Recent Advances, Proceedings of the 9th International Conference, The Institute of Sound and Vibration Research, University of Southampton, Paper 129, Southampton, UK, 2006.

14

American Institute of Aeronautics and Astronautics 